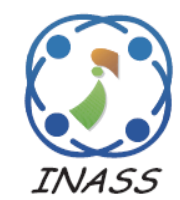

http://www.inass.org/

\title{
Grey Wolf Optimizer with Linear Collaborative Discriminant Regression Classification based Face Recognition
}

\author{
Sangamesh Hosgurmath ${ }^{1 *} \quad$ Viswanatha Vanjre Mallappa $^{2}$ \\ ${ }^{1}$ Visvesvaraya Technological University, Belagavi., India \\ ${ }^{2}$ Sarojini Liladharan Nair College of Engineering, Raichur, India \\ * Corresponding author's Email: Sangu_hosmat@yahoo.com
}

\begin{abstract}
For the past few decades, biometric Face Recognition (FR) is the active research area in different domains such as image processing, pattern recognition, etc. The existing FR system has several limitations such as Single Sample Problem, maximum Reconstruction Errors (REs), these problems decreases the FR rate. In this research paper, an efficient FR method is proposed, namely Grey Wolf Optimizer based Linear Collaborative Discriminant Regression Classification (GWO-LCDRC). The optimization technique of GWO algorithm is applied in LCDRC to select the relevant weight value in LCDRC. For every training sample, optimal weight values are selected to improve the recognition rate. The proposed GWO-LCDRC method maximize the collaboration of Between Class RE (BCRE) and minimize the Within Class RE (WCRE). An experimental analysis conducted on the two standard facial databases namely ORL and YALE. The proposed GWO-LCDRC method improved the performance of recognition accuracy with respect to different training samples. Also, the performance is compared with the traditional methods Linear Regression Classification (LRC), Linear Discriminant Regression Classification (LDRC), and LCDRC. The overall experiment demonstrated that the proposed GWO-LCDRC method achieved approximately $3 \%$ and $6.5 \%$ of FR accuracy improvement with respect to ORL and YALE respectively.
\end{abstract}

Keywords: Face recognition, Grey wolf optimizer, Linear collaborative discriminant regression classification, Linear discriminant regression classification, Linear regression classification.

\section{Introduction}

Now-a-days, biometric FR is more emerging research area in the image processing field. The main objective of biometry is to detect the people from several observable characteristics such as their face, fingerprints, iris, etc. These traits are suitable for biometric recognition, but human FR provides more security for person authentication [1, 2]. Hence, FR is the very popular research area and widely used in different applications such as criminal identification, access control system, information security, etc. [3, 4]. But, FR is the difficult task for computer-based systems. There are two types of FR system, those are Appearance-based system [5] and Feature-based system [6]. The image of face is captured from the unconstrained conditions such as variations in poses, expressions, illuminations, etc. The existing FR algorithms still failed to fulfill the real applications because of severe challenges [7-8]. Numerous existing researchers used several methods such as Fisher Liner Discriminant [9], Particle Swarm Optimization [10], Linear Regression Classification [11], etc. for the FR.

Many traditional FR systems have several limitations like low quality of images due to variations in lighting and occlusion, high dimensional images which leads to computational difficulty and expensive time cost [12], single sample problem (only on training sample used for each object) [13], etc. In this research paper, an efficient FR method is proposed namely GWO-LCDRC. The significant contribution is mentioned below.

- The LCDRC used different weight to describe the various training sets and suitable weight value is selected by GWO algorithm. 
- The selection of optimal weighted value effectively reduces the BCRE and WCRE.

- The proposed algorithm is applied to the standard facial database such as ORL, YALE for improving the recognition rate and decrease the RE.

This paper is organized as follows. Section 2 reviews numerous recent research papers on FR strategies. Section 3 describes the problem statement and solutions. In section 4, an effective optimization technique based LCDRC namely (GWO-LCDRC) is described. Section 5 shows a comparative experimental result for proposed and existing FR strategies with respect to standard datasets: ORL and YALE. The conclusion is made in section 6.

\section{Literature review}

This section describes a few recent research approaches suggested by the researchers on FR system. A brief evaluation of some essential contributions to the existing literatures is presented in this section.

R. Senthilkumar, and R. K. Gnanamurthy, [14] proposed Documentation-based approach of Bag-OfVisual-Words (BOVW) method for facial image recognition. In BOVW method, relevant facial features were extracted from the Scale Invariant Feature Transform (SIFT) descriptor and extracted features were input to the Support Vector Machine (SVM) classifier. The major contribution of this proposed method was to improve the FR rate and decreases the recognition time. An experimental analysis demonstrated that $50 \%$ of training and $50 \%$ of testing were performed in standard facial datasets. The main disadvantage in BOVW method is that it requires very large feature extraction time to train $50 \%$ of samples.

S. P. Ramalingam and C. M. P. V. S. Sita, [15] presented Dimensionality Reduction method namely Local Directional Number Pattern (DR-LDNP) for facial expression recognition. The DR-LDNP feature descriptor extracted the intensity variation as well as facial textural features from the input image. The proposed descriptor extremely decreased the dimensionality of the feature vectors and improved the efficiency of the recognition system. This improved class variance as well as reduced the within class variance. The proposed feature descriptor improved the recognition accuracy with respect to different complexities such as lighting conditions, various pose, and random noise. In JAFFEE database, the recognition rate was comparatively less because it included inaccurate data, hence it influenced the training and testing performance.
P. Huang, G. Gao, C. Qian, G. Yang, and Z. Yang, [16] proposed an efficient FR method namely Fuzzy Linear Regression Discriminant Projection (FLRDP). At first, FLRDP calculates every sample's membership degree to corresponding classes and with the help of membership degree information to the fuzzy BCRE and WCRE were generated via Fuzzy K Nearest Neighbor (FKNN). Experiment was conducted for FLRDP on ORL, FERET, and CMU PIE facial database and demonstrated the FLRDP algorithm achieved robust result when large variation in illumination, facial poses and expressions. Even though the method FLRDP suffered from the parameter selection problem. It was also found that the parameter $k$ had an impact on the performance of FLRDP.

Z.H.A.O. Jian, Z.H.A.N.G. Chao, Z.H.A.N.G. Shunli, L.U. Tingting, S.U. Weiwen, and J.I.A. Jian, [17] proposed two significant approaches for FR such as Facial Pose Pre-Recognition (FPPR) as well as Dual-Dictionary Sparse Representation Classification (DD-SRC). At first, FPPR approach detected the facial feature points and separate the test samples from the input. After that, DD-SRC approach detected the most similar features in training samples and reduced the mutual interference between the different poses. The proposed model achieved better performance in large size datasets. Here, the number of facial images was limited in database, hence FPPR may fail to detect the feature points accurately.

S. Biswas and J. Sil, [18] developed a new method for FR to increase the recognition rate specifically Contourlet Transform (CNT) and Curvelet Transform (CLT) methods. The proposed method provided two advantages such as (i) extract the highly correlated information on statistical features of different directional sub bands by CNT. (ii) The edge or curve of the images was effectively represented by CLT. These two major functionalities of the proposed method improved the overall FR performance. Threshold method was employed for more relevant sub-bands selection, but it takes more time for sub band selection.

An optimization based GWO with LCDRC method is implemented for improving the performance of FR rate and to rectify the above limitations.

\section{Problem statement}

This section describes about the problem statement of the existing linear regression classification based different methods of FR system. 
Also detailed about how the proposed methodology gives solution to the described problems.

- The traditional LRC algorithm used the least square algorithm for solving the linear regression problem. The LRC can't perform in variations of image brightness and not able to classify the samples that distributed around intersections [19].

- The LRC algorithm was used in FR system, but a major problem in LRC is multicollinearity. When each class includes limited training samples, all classes share the residuals with other classes. The query sample not perfectly represented in limited samples, hence LRC failed to satisfy the classification performance [20-21].

- The LDRC method was used to find the linear subspace in LRC in order to improve performance of recognition. The LRDC was used labeled training data to construct the BCRE and WCRE. But, LDRC provided equal priority for each class sample which may lead to suboptimal solution of LDRC for discriminative feature extraction [22].

- The traditional LCDRC algorithm was used for rectifying the problem of class-specific BCRE domination issue. But, least square estimation of projection of similarity matrix depends on the regression line, therefore the residual error may increase [23-24].

Solution: To overcome above mentioned drawback, optimization based LCDRC algorithm of FR system is implemented for improving the performance of BCRE and WCRE. Here, a new optimization algorithm is used, namely GWO for selecting the optimal weight value in LCDRC. In LCDRC, for calculating the distances the WC feature compared with the total number of class features. Due to GWO-LCDRC, the ratio of the distance between the classes maximized extremely and the distance of within class features reduce significantly. The GWO algorithm selects the best weight value in LCDRC to reduce the RE. As a result, the proposed method finds a discriminant subspace by maximizing the ratio of BCRE and decrease the WCRE simultaneously. The detailed description about GWO with LCDRC is given in section 4 .

\section{Proposed methodology}

The proposed FR approach is utilized to analyze the human facial images by using LCDRC and it used the GWO technique for selecting best weighted value in order to improve recognition rates. The swarm intelligent methods are usually employed to solve the optimization problems that can't have the leader to monitor the entire proceeding period. This problem is rectified by GWO method because it consists of individual leadership capacity. Hence, GWO with the classification criteria improves the LCDRC algorithm as GWO-LCDRC algorithm in order to increase the proportion of BCRE over WCRE for calculating the weighted value in LCDRC with the help of the GWO. The following section describes the LRC, LDRC, LCDRC and proposed GWO-LCDRC methods based on FR.

\subsection{Linear regression classification}

Consider a total number of classes indicated as $N$ and training samples are indicated as $q_{m}$ for $m^{\text {th }}$ class, $m=1,2, \ldots . N$. An individual training sample is converted to a vector via column concatenation. The dimension of the vector is represented as $p$ and class specific model is indicated as $Y_{m}$ includes p-dimensional image vectors and it's mathematically shown in the Eq. (1).

$$
Y_{m}=\left[w_{m}^{1}, w_{m}^{2}, \ldots w_{m}^{q_{m}}\right] \in \mathbb{R}^{p \times q_{m}}
$$

Whereas, $w_{m}^{q_{m}}$ is the $q_{m}$ training sample from the class $m$, and unlabeled probe image converted into image vector and it's denoted as $X$. Also, $X$ is included each class specific model and shown in the Eq. (2).

$$
X=Y_{m} \beta_{m} m=1,2, \ldots N
$$

The $\beta_{m} \in \Re^{q_{m} \times 1}$ is denoted as regression parameter, $\beta_{m}$ is evaluated by employing the least square estimation. Mathematically, $\beta_{m}$ is described in the Eq. (3).

$$
\hat{\beta}_{m}=\left(Y_{m}^{T} Y_{m}\right)^{-1} Y_{m}^{T} X
$$

Whereas, $T$ is indicated as transformation of the classes. Then the response from each class $m$ are estimated in Eq. (4),

$$
\hat{x}=Y_{m} \hat{\beta}_{m}=Y_{m}\left(Y_{m}^{T} Y_{m}\right)^{-1} Y_{m}^{T} X, m=1,2, \ldots . N
$$

The $Y_{m}\left(Y_{m}^{T} Y_{m}\right)^{-1} Y_{m}^{T}$ is replayed as $H_{i}$ hence, it's shown in the Eq. (5),

$$
\hat{X}=H_{i} X
$$


Whereas, $H_{i}$ is indicated as hat matrix that plots $X$ into $\widehat{X}_{m}$. At last, the reconstruction error of each class is evaluated with lowest reconstruction error, which is mathematically denoted in the Eq. (6).

$$
e_{m}(X)=\left\|X-\hat{X}_{m}\right\|_{2} m=1,2, \ldots N
$$

The major limitation of LRC algorithm is that it can't perform in a limited number of training samples, hence the recognition performance decreased. For this reason, LDRC algorithm is used. The following section describes the LDRC algorithm.

\subsection{Linear discriminant regression classification}

The LDRC method is used to find the efficient discriminant subspace for LRC to maximize the BCRE and minimize the WCRE. If the Reconstruction Error (RE) of the true class is maximized and the RE of false class is minimized. The projection matrix is mathematically shown in the Eq. (7).

$$
\max _{P}=\max _{P}\left(\frac{R E_{B C}}{R E_{W C}}\right)
$$

Whereas, $P$ is indicated as projection matrix and the RE of between class and within class is indicated as BC and WC respectively. After calculation of $P$, total number of training samples and probe images are converted to the subspace $X=P^{T} Y$. Then, the BCRE and WCRE are represented in inter-class and intra-class variances of the training samples that are denoted in Eq. (8) and (9).

$$
W C R E=\frac{1}{n} \sum_{m=1}^{N}\left(Y_{m}-Y_{m}^{\text {intra }}\right)\left(Y_{m}-Y_{m}^{\text {intra }}\right)^{T}
$$

$$
\begin{gathered}
\text { BCRE }=\frac{1}{n(m-1)} \sum_{m=1 n=1}^{n} \sum_{n \neq a\left(Y_{m}\right)}^{m}\left(Y_{m}-\right. \\
\left.Y_{m n}^{\text {inter }}\right)\left(Y_{m}-Y_{m}^{\text {inter }}\right)^{T}
\end{gathered}
$$

Where, inter and intra-classes are determined as $Y_{m n}^{i n t e r}$ and $Y_{m}^{i n t r a}, T$ is signified as transformation of the classes. The LDRC algorithm provides equal priority for interclass and intra class elements, hence the optimal solution selection performance may degrade. LCDRC algorithm is explained in the following section.

\subsection{Linear collaborative discriminant regression classification}

The LCDRC algorithm rectifies the problem of class-specific BCRE domination issue with the help of collaborative representation. In LCDRC, the WCRE features are compared with the all class features to measure the distance. Due to GWOLCDRC, the ratio of distance between the classes maximize extremely and the distance of within class features reduce significantly. In WCRE, individual features of the class compensate with the $n$ number of class features. The connection between the WCRE and CBCRE is shown in the Eq. (10) and (11).

$$
\begin{gathered}
\text { WCRE }=\frac{1}{n} \sum_{n=1}^{m} \sum_{a=1}^{N} \| P^{T} Y_{m n}- \\
P^{T} Y_{m n}^{\text {intra }} \beta_{m n}^{\text {intra }} \|_{2}^{2} \\
\text { CBCRE }=\frac{1}{n} \sum_{n=1}^{m} \sum_{a=1}^{N} \| P^{T} Y_{m n}- \\
P^{T} Y_{m n}^{\text {inter }} \beta_{m n}^{\text {inter }} \|_{2}^{2}
\end{gathered}
$$

Re-written Eq. (10) and (11) are shown in Eq. (12) and (13).

$$
\begin{gathered}
\text { WCRE }=\frac{1}{n} \sum_{n=1}^{m} \sum_{a=1}^{n}\left(Y_{m n}-\right. \\
\left.Y_{m n}^{\text {intra }} \beta_{m n}^{\text {intra }}\right)^{T} P P^{T}\left(Y_{m n}-Y_{m n}^{\text {intra }} \beta_{m n}^{\text {intra }}\right)^{T} \\
\text { CBCRE }=\frac{1}{n} \sum_{n=1}^{m} \sum_{a=1}^{n}\left(Y_{m n}-\right. \\
\left.Y_{m n}^{\text {inter }} \beta_{m n}^{\text {inter }}\right)^{T} P P^{T}\left(Y_{m n}-Y_{m n}^{\text {inter }} \beta_{m n}^{\text {inter }}\right)^{T}
\end{gathered}
$$

In Eq. (12) and Eq. (13) the factor $\frac{1}{n}$ is available in both CBCRE and WCRE, hence $\frac{1}{n}$ is possible to remove using algebraic deduction method and it's not affecting the value of CBCRE and WCRE. It's mathematically shown in the Eq. (14) and (15).

$$
\begin{gathered}
\text { WCRE }=\sum_{n=1}^{m} \sum_{a=1}^{n} \operatorname{tr}\left(P ^ { T } \left(Y_{m n}-\right.\right. \\
\left.\left.Y_{m n}^{\text {intra }} \beta_{m n}^{\text {intra }}\right)\left(Y_{m n}-Y_{m n}^{\text {intra }} \beta_{m n}^{\text {intra }}\right)^{T} P\right) \\
\text { CBCRE }=\sum_{n=1}^{m} \sum_{a=1}^{n} \operatorname{tr}\left(P ^ { T } \left(Y_{m n}-\right.\right. \\
\left.\left.Y_{m n}^{\text {inter }} \beta_{m n}^{\text {inter }}\right)\left(Y_{m n}-Y_{m n}^{\text {inter }} \beta_{m n}^{\text {inter }}\right)^{T} P\right)
\end{gathered}
$$

In Eq. (14) and Eq. (15), the tr (.) is denoted as trace operator and finally the WCRE and BCRE is represented in Eq. (16) and (17).

$$
\begin{gathered}
\text { WCRE }=\sum_{n=1}^{m} \sum_{a=1}^{n}\left(Y_{m n}-\right. \\
\left.Y_{m n}^{\text {intra }} \beta_{m n}^{\text {intra }}\right)\left(Y_{m n}-Y_{m n}^{\text {intra }} \beta_{m n}^{\text {intra }}\right)^{T} \\
\text { CBCRE }=\sum_{n=1}^{m} \sum_{a=1}^{n}\left(Y_{m n}-\right. \\
\left.Y_{m n}^{\text {inter }} \beta_{m n}^{\text {inter }}\right)\left(Y_{m n}-Y_{m n}^{\text {inter }} \beta_{m n}^{\text {inter }}\right)^{T}
\end{gathered}
$$




\subsection{Grey wolf optimization based linear collaborative discriminant regression classification}

In GWO, the optimization is done by metaheuristic techniques, which is bio-inspired from nature of grey wolves. In a grey wolf community, there are four categories of grey wolves namely alpha, beta, delta, and omega. Among them, alpha is considered as the leader of the group. Beta wolves assist alpha in decision making and hunting which are considered to be the next candidate eligible to the alpha if alpha attains the stage of retirement or death while hunting. Delta wolves or alpha wolves that protect the boundaries of their group. Omega wolves are the least prioritized wolves, that need to submit all the other wolves and follow all other category wolves.

Assume that $w_{i}=\left\{w_{i 1}, w_{i 2} \ldots w_{i n}\right\}$ represents position vectors in the search space, whereas the dimension of the problem is shown as $n$. The fitness function (based on problem definition) is employed to estimate the position of the wolves. Based on the fitness value the best wolves are classified as the first solution that is represented as $\alpha$, the second is $\beta$, and the third is $\delta$ respectively. In the best solution searching process, the wolves update their position according to the position of $\alpha, \beta$ and $\delta$.

In the starting stage, the wolf population is generated and the position of every wolf is initialized. The co-efficient vectors of $\vec{A}$ and $\vec{C}$ are described in Eq. (18) and (19).

$$
\begin{aligned}
& \vec{A}=2 \vec{a} \cdot \overrightarrow{r 1}-\vec{a} \\
& \vec{C}=2 \overrightarrow{r 2}
\end{aligned}
$$

The $\vec{A}$ takes random values in the range of $[-a, a]$, $\vec{C}$ with a random value in the range $[0,2]$ and it avoids the trap of local optimal. After the initialization of the coefficients, every wolf (search agent) fitness value is estimated. After that, best fitness solutions are selected as first, second and third such as $\alpha, \beta$ and $\delta$ respectively.

$$
\begin{gathered}
\left(\vec{D}_{\alpha}\right)=\left|\vec{C}_{1} \cdot \vec{X}_{\alpha}-\vec{X}\right|,\left(\vec{D}_{\beta}\right)=\mid \vec{C}_{2} \cdot \vec{X}_{\beta}- \\
\vec{X}\left|,\left(\vec{D}_{\delta}\right)=\right| \vec{C}_{3} \cdot \vec{X}_{\delta}-\vec{X} \mid \\
\vec{X}_{1}=\vec{X}_{\alpha}-\vec{A}_{1} \cdot\left(\vec{D}_{\alpha}\right), \vec{X}_{2}=\vec{X}_{\beta}-\vec{A}_{2} \cdot\left(\vec{D}_{\beta}\right), \vec{X}_{3}= \\
\vec{X}_{\delta}-\vec{A}_{3} \cdot\left(\vec{D}_{\delta}\right) \\
\vec{x}_{(t+1)}=\frac{\vec{X}_{1}+\vec{X}_{2}+\vec{X}_{3}}{3}
\end{gathered}
$$

In each iteration of the algorithm, the wolf's position update based on the position of wolves such as $\alpha, \beta$ and $\delta$. In addition, values of vectors $\vec{A}_{1}, \vec{C}$ and $\vec{a}$ are updated. On the basis of new positions, the value of the fitness function of wolves is calculated and $\alpha, \beta$ and $\delta$ will be selected. In Eq. (22), the $\alpha, \beta$, and $\delta$ values and their positions are updated. These updated values forward to the alpha, beta and delta, again iterations are repeated. The calculation of best weighted value is shown in the Eq. (23).

$$
X=\max (x(t))
$$

Whereas, $X$ is the best optimal solution with respect $x(t)$ and it indicate the number of iterations. The $t$ is indicated as number of iterations $(\mathrm{t}=500)$. After calculation of best solution $X$ value is applied to the LCDRC algorithm to select the best weighted value. The LCDRC calculation is shown in the Eq. (16) and (17). The GWO based LCDRC calculation is shown in the Eq. (24) and (25).

$$
\begin{gathered}
\text { WCRE }=\frac{1}{n} \sum_{n=1}^{m} \sum_{a=1}^{n} X\left(Y_{m n}-\right. \\
\left.Y_{m n}^{\text {intra }} \beta_{m n}^{\text {intra }}\right)\left(Y_{m n}-Y_{m n}^{\text {intra }} \beta_{m n}^{\text {intra }}\right)^{T} \\
\text { CBCRE }=\frac{1}{n} \sum_{n=1}^{m} \sum_{a=1}^{n} X\left(Y_{m n}-\right. \\
\left.Y_{m n}^{\text {inter }} \beta_{m n}^{\text {inter }}\right)\left(Y_{m n}-Y_{m n}^{\text {inter }} \beta_{m n}^{\text {inter }}\right)^{T}
\end{gathered}
$$

Whereas, $X$ is represented as the best weighted value in the LCDRC. The search agents of the proposed GWO algorithm update their positions with respect to the alpha, beta, and delta locations. The constraint handling method is assigned objective function value. If they violate any of the constraints, then it automatically replaced with a new search agent in the next iteration. The objective function value is in the range of $[-1,1]$. Hence, GWO select the best weighted value in LCDRC for FR. The proposed GWO-LCDRC algorithm significantly maximize the BCRE and reduces the WCRE.

\section{Experimental result and discussion}

In this section, the experimental outcome is described in detailed, which is implemented in PC with $1.8 \mathrm{GHz}$ Pentium IV processor using MATLAB (version 6.5). To evaluate the effectiveness of the proposed algorithm, the performance of LCDRC is compared with GWO-LCDRC on the reputed face database sets like ORL, and YALE. In experiment, all the facial images are cropped at the size of $32 \times$ 32. 


\subsection{Dataset description}

For examining the performance of the proposed system, three extensively applied datasets: ORL and Yale B are used. The detailed description about the acquired datasets is described below.

\subsubsection{ORL dataset}

In this experimental analysis, ORL database is used to acquire the input facial image. This database includes 400 face images of 40 persons with 10 different expressions. The face images were taken under different light conditions and with different facial expressions. The ORL sample data is taken from

https://www.cl.cam.ac.uk/research/dtg/attarchive/fac edatabase.html and shown in Fig. 1.

\subsubsection{YALE dataset}

The YALE facial database consists of 165 face images of 15 persons. For each person, 15 images are collected under various expressions. All of the face images are cropped into a size of $32 \times 32$. The YALE sample dataset is taken from http://vision.ucsd.edu/content/yale-face-database and shown in Fig. 2.

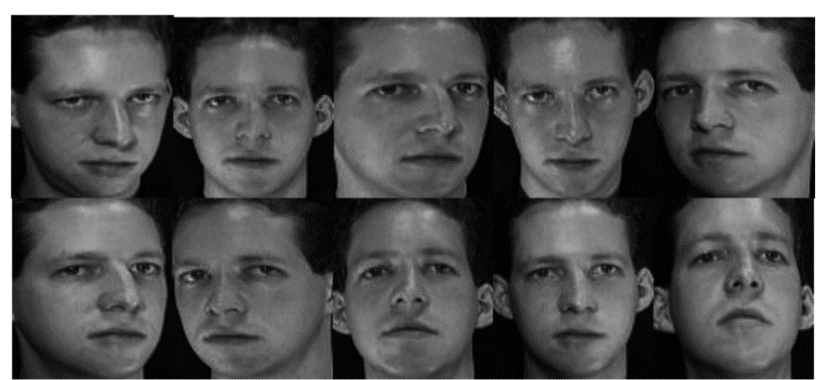

Figure. 1 Sample of ORL database

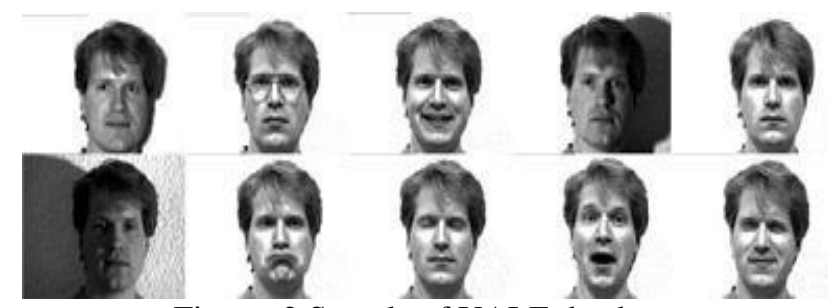

Figure. 2 Sample of YALE database

\subsection{Experimental analysis of ORL database}

In this section, a facial image recognition based Without GWO-LCDRC method and with GWOLCDRC method performance is analyzed with respect to the ORL database. For experiments, the training data are selected from the database such as Two training (2T), Three Training (3T), Four Training (4T), Five Training (5T), Six Training (6T), Seven Training (7T) and Eight Training (8T). Each experiment is repeated 50 times \& the average result is reported. According to the Table 1, the performance of accuracy of the different training samples of with GWO-LCDRC and without GWOLCDRC method is evaluated. The experimental performance of the ORL database and four training sample of ORL database is graphically shown in Fig. 3.

Fig. 3 represents the performance of the four training samples with respect to the ORL database. According to the figure, $\mathrm{X}$-axis is indicated as different dimensions of the samples and the $\mathrm{Y}$-axis is represented as accuracy performance (\%). Compared to the $2 \mathrm{~T}$ and $3 \mathrm{~T}$, the $4 \mathrm{~T}$ sample performance gradually increased. The recognition performance of with GWO-LCDRC method shows better results than the without GWO-LCDRC. In four training sample, with GWO-LCDRC method achieved $93.55 \%$ of recognition accuracy and without GWO-LCDRC method achieved $92.14 \%$ of accuracy.

Table 1. Performance analysis of ORL database

\begin{tabular}{|c|c|c|c|c|c|c|c|c|}
\hline \multirow{2}{*}{$\begin{array}{c}\text { Facial } \\
\text { Database }\end{array}$} & \multirow{2}{*}{ Methods } & \multicolumn{6}{|c|}{ Performance Evaluation - Accuracy (\%) } \\
\cline { 3 - 8 } & & \multicolumn{7}{|c|}{ Number of training Samples } \\
\cline { 3 - 8 } & & $\begin{array}{c}\text { Two } \\
\text { Training } \\
\text { Samples }\end{array}$ & $\begin{array}{c}\text { Three } \\
\text { Training } \\
\text { Samples }\end{array}$ & $\begin{array}{c}\text { Four } \\
\text { Training } \\
\text { Samples }\end{array}$ & $\begin{array}{c}\text { Five } \\
\text { Training } \\
\text { Samples }\end{array}$ & $\begin{array}{c}\text { Six } \\
\text { Training } \\
\text { Samples }\end{array}$ & $\begin{array}{c}\text { Seven } \\
\text { Training } \\
\text { Samples }\end{array}$ & $\begin{array}{c}\text { Eight } \\
\text { Training } \\
\text { Samples }\end{array}$ \\
\cline { 2 - 9 } & $\begin{array}{c}\text { Without GWO- } \\
\text { LCDRC }\end{array}$ & 85.1 & 89.61 & 92.14 & 94.89 & 96.5 & 96.01 & 98.0 \\
\cline { 2 - 9 } & $\begin{array}{c}\text { With GWO- } \\
\text { LCDRC }\end{array}$ & 89.37 & 92.79 & 93.55 & 95.11 & 97.01 & 97.37 & 98.5 \\
\hline
\end{tabular}




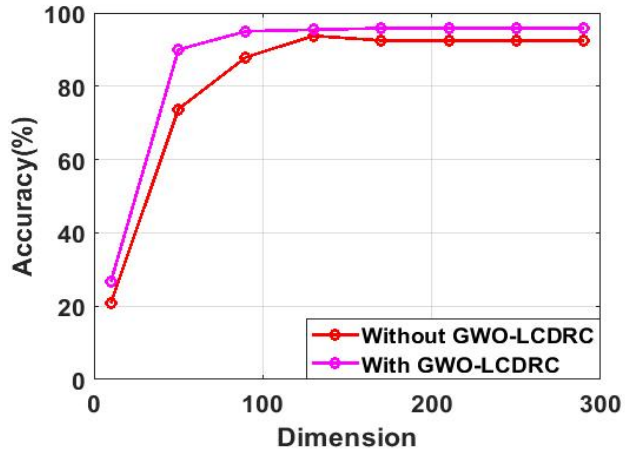

Figure. 3 Performance of four training sample

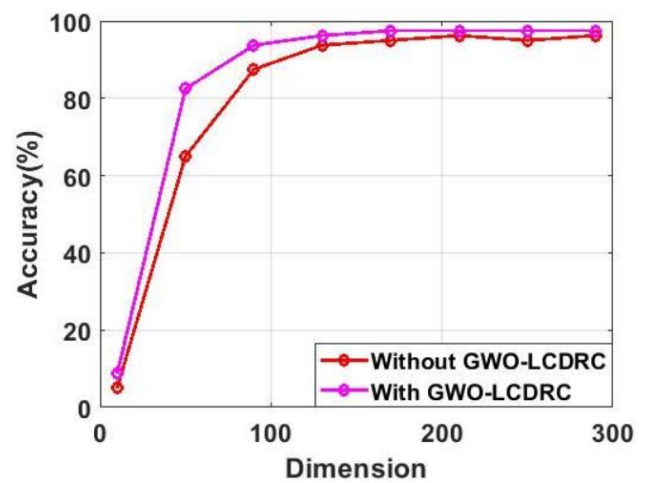

Figure. 4 Performance of eight training sample
Fig. 4 indicates the accuracy performance of the eight training sample with respect to ORL database. In training class, the recognition performance is measured with respect to corresponding feature dimension. In eight training classes, overall 50 iterations are performed and one of the iteration sample image is shown in the Fig. 4. The without GWO-LCDRC algorithm attained $98.0 \%$ and with GWO-LCDRC algorithm achieved $98.5 \%$ of recognition accuracy.

\subsection{Experimental analysis of YALE database}

This section demonstrates the facial recognition performance of with GWO-LCDRC method and without GWO-LCDRC method with respect to different training classes of YALE database. In the training set, if a number of training samples varies, then recognition rate also varies. Here, 50 iterations for training and testing samples for FR has been considered. Performance of minimum four training samples and maximum eight training sample's performances are shown in Fig. 5 and Fig. 6. The Table 2 shows the performance evaluation of proposed and existing FR methods of YALE database.

Table 2. Performance analysis of YALE database

\begin{tabular}{|c|c|c|c|c|c|c|c|c|}
\hline \multirow[t]{2}{*}{$\boldsymbol{\mu}$} & \multirow[t]{3}{*}{ Methods } & \multicolumn{7}{|c|}{ Performance Evaluation - Accuracy (\%) } \\
\hline & & \multicolumn{7}{|c|}{ Number of training Samples } \\
\hline \multirow{3}{*}{ YALE } & & $\begin{array}{c}\text { Two } \\
\text { Training } \\
\text { Samples }\end{array}$ & $\begin{array}{c}\text { Three } \\
\text { Training } \\
\text { Samples }\end{array}$ & $\begin{array}{c}\text { Four } \\
\text { Training } \\
\text { Samples }\end{array}$ & $\begin{array}{c}\text { Five } \\
\text { Training } \\
\text { Samples }\end{array}$ & $\begin{array}{c}\text { Six } \\
\text { Training } \\
\text { Samples }\end{array}$ & $\begin{array}{c}\text { Seven } \\
\text { Training } \\
\text { Samples }\end{array}$ & $\begin{array}{c}\text { Eight } \\
\text { Training } \\
\text { Samples }\end{array}$ \\
\hline & $\begin{array}{l}\text { Without GWO- } \\
\text { LCDRC }\end{array}$ & 67.97 & 72.12 & 74.80 & 79.59 & 82.51 & 86.16 & 87.82 \\
\hline & $\begin{array}{l}\text { With GWO- } \\
\text { LCDRC }\end{array}$ & 65.82 & 72.73 & 76.40 & 79.89 & 83.47 & 87.20 & 89.6 \\
\hline
\end{tabular}

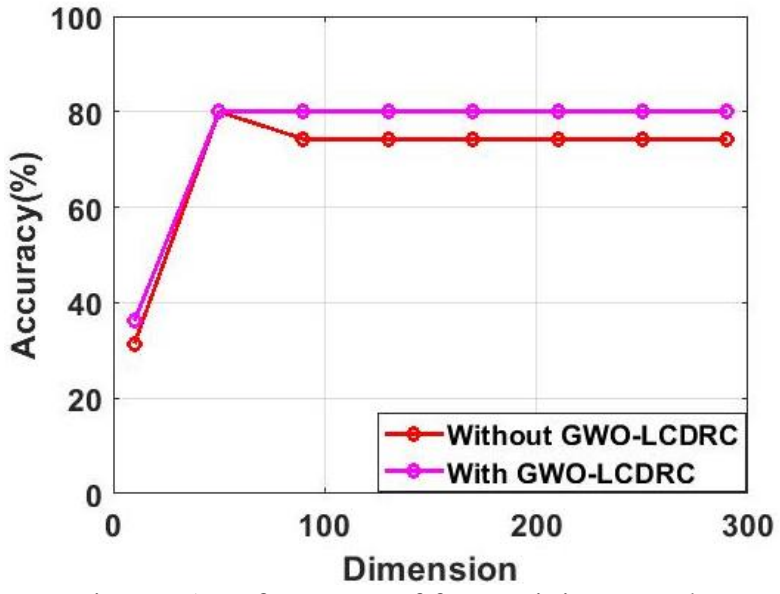

Figure. 5 Performance of four training sample
Fig. 5 represents the accuracy performance of 4T training samples of YALE database. The recognition performance is measured with respect to corresponding dimensions of features. In four training classes, without GWO-LCDRC method achieved $74.80 \%$ of recognition accuracy and with GWO-LCDRC method achieved $76.40 \%$ of accuracy. Compare to the without GWO-LCDRC, the proposed with GWO-LCDRC method shown better results. The GWO algorithm selects the best weight value for different training classes in order to reduce the intra and inter class RE.

Fig. 6 depicts the performance of the eight training samples with respect to YALE database. Compared to the $4 \mathrm{~T}$ performance, the $8 \mathrm{~T}$ facial recognition performance improved. The recognition 


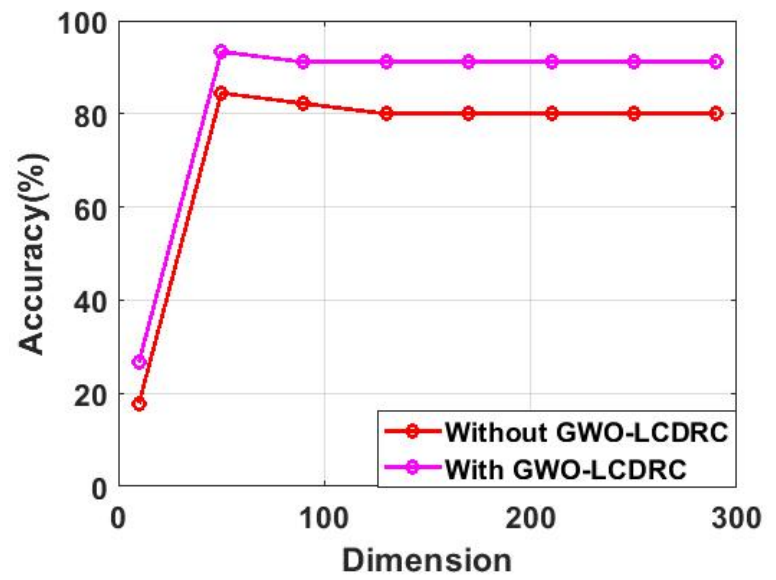

Figure. 6 Performance of eight training sample

Table 3. Comparative Analysis

\begin{tabular}{|c|c|c|c|}
\hline \multirow{2}{*}{$\begin{array}{l}\text { Author } \\
\text { Name }\end{array}$} & \multicolumn{3}{|c|}{ Recognition Accuracy (\%) } \\
\hline & Methodology & $\begin{array}{c}\text { ORL } \\
\text { Database }\end{array}$ & $\begin{array}{c}\text { YALE } \\
\text { Database }\end{array}$ \\
\hline $\begin{array}{c}\text { Y. Peng, } \\
\text { [19] }\end{array}$ & ILRC & 90.58 & - \\
\hline \multirow{3}{*}{$\begin{array}{c}\text { X. Qu, S. } \\
\text { Kim, R. Cui, } \\
\text { and H. J. } \\
\text { Kim [23] }\end{array}$} & LRC & 88.87 & 61.62 \\
\hline & LDRC & 89.83 & 66.48 \\
\hline & LCDRC & 94.21 & 77.05 \\
\hline \multirow{2}{*}{$\begin{array}{c}\text { J. Wang, C. } \\
\text { Lu, M. } \\
\text { Wang, P. Li, } \\
\text { S. Yan, and } \\
\text { X. Hu [24] }\end{array}$} & LSRC & 94.0 & 78.40 \\
\hline & ASRC & 95.85 & 83.17 \\
\hline $\begin{array}{c}\text { Proposed } \\
\text { Work }\end{array}$ & $\begin{array}{c}\text { GWO- } \\
\text { LCDRC }\end{array}$ & 98.5 & 89.6 \\
\hline
\end{tabular}

rate is constant after reaching the corresponding feature dimension. Without GWO-LCDRC method achieved $87.82 \%$ of recognition accuracy and with GWO-LCDRC method achieved $89.6 \%$ of accuracy.

\subsection{Comparative study}

In this section, comparative study of existing and proposed FR method is shown in Table 3. X. Qu, S. Kim, R. Cui, and H. J. Kim [23] proposed LCDRC algorithm was used to calculate the BCRE. The CBCRE increases the particular class BCRE and highlights the particular class RE. In an experimental analysis, LRC, LDRC, and LCDRC method recognition rate were calculated with respect to ORL and YALE database. J. Wang, C. Lu, M. Wang, P. Li, S. Yan, and X. Hu [24] proposed Adaptive Sparse Representation based Classification (ASRC) for FR. The ASRC method uses the sparsity and selects the most discriminant samples. Also, ASRC results were compared with the existing Locality-constrained Sparse Representation based Classifier (LSRC) with respect to ORL and YALE database in order to estimate the recognition accuracy. Y. Peng, [19] presented an effective FR system, namely ILRC to estimate the RE in BC and WC. The ILRC method simultaneously considers the testing samples and training samples of RE. Compared to the existing methods, the proposed methods shown better facial recognition rate.

Finally, the proposed GWO-LCDRC algorithm achieved recognition rate of $98.5 \%$ and $89.6 \%$ with respect to ORL dataset and YALE dataset respectively. The proposed LCDRC method use the GWO optimizer for select the optimal weight value in order to decrease the intra and inter class errors.

\section{Conclusion}

FR is the challenging research area in the field of biometrics. In this research paper, the GWO-LCDRC method is proposed to improve the FR rate. The GWO algorithm selects the efficient weighted values and forward to the LCDRC algorithm for FR. The proposed GWO-LCDRC algorithm maximize the BCRE and minimize the WCRE in training sets. An experimental analysis is conducted on two publicly available databases such as ORL and YALE. The performance of facial recognition is measured by two efficient methods such as with GWO-LCDRC and without GWO-LCDRC with respect to Accuracy parameter. The GWO-LCDRC algorithm achieved 98.5\% and $89.6 \%$ of FR accuracy in terms of ORL and Yale dataset respectively. In future, the research work can be extended as an efficient Deep Learning recognition technique can be used to improve the recognition rate and reduce the RE.

\section{References}

[1] V. Štruc, and N. Pavešić, "The complete gaborfisher classifier for robust face recognition", EURASIP Journal on Advances in Signal Processing, Vol.1, pp.847680, 2010.

[2] R. Sharma and M. S. Patterh, "A new hybrid approach using PCA for pose invariant face recognition", Wireless Personal Communications, Vol.85, No.3, pp.1561-1571, 2015.

[3] W. Xu and E. J. Lee, "A hybrid method based on dynamic compensatory fuzzy neural network algorithm for face recognition", International Journal of Control, Automation and Systems, Vol.12, No.3, pp.688-696, 2014.

[4] H. Mliki, E. Fendri, and M. Hammami, "Face recognition through different facial expressions", Journal of Signal Processing Systems, Vol.81, No.3, pp.433-446, 2015. 
[5] R. Senthilkumar and R. K. Gnanamurthy, "A robust wavelet based decomposition of facial images to improve recognition accuracy in standard appearance based statistical face recognition methods", Cluster Computing, pp.110, 2018.

[6] C. Singh, N. Mittal, and E. Walia, "Complementary feature sets for optimal face recognition", EURASIP Journal on image and video Processing, Vol.1, pp.35, 2014.

[7] Y. Li, W. Zheng, Z. Cui, and T. Zhang, "Face recognition based on recurrent regression neural network", Neurocomputing, Vol.297, pp.50-58, 2018.

[8] J. Wang, C. Lu, M. Wang, P. Li, S. Yan, and X. $\mathrm{Hu}$, "Robust face recognition via adaptive sparse representation", IEEE Transactions on Cybernetics, Vol.44, No.12, pp.2368-2378, 2014.

[9] Z. Xu, Y. Jiang, Y. Wang, Y. Zhou, W. Li, and Q. Liao, "Local Polynomial Contrast Binary Patterns for "Face Recognition", Neurocomputing, In Press, 2018.

[10] S. A. Khan, M. Ishtiaq, M. Nazir, and M. Shaheen, "Face recognition under varying expressions and illumination using particle swarm optimization", Journal of Computational Science, Vol.28, pp.94-100, 2018.

[11] Y. Zhu, C. Zhu, and X. Li, "Improved principal component analysis and linear regression classification for face recognition", Signal Processing, Vol.145, pp.175-182, 2018.

[12] Y. Li and C. Qi, "Face recognition using position-dictionaries and region covariance feature", Signal, Image and Video Processing, Vol.8. No.6, pp.1139-1147, 2014.

[13] C. H. Hu, M. J. Ye, W. L. Zeng, and X. B. Lu, "An adaptive approximation image reconstruction method for single sample problem in face recognition using FLDA", Multimedia Tools and Applications, Vol. 74, No.23, pp.10313-10334, 2015.

[14] R. Senthilkumar and R. K. Gnanamurthy, "A proposed method for the improvement in biometric facial image recognition using document-based classification", The Journal of Supercomputing, pp.1-19, 2018.

[15] S. P. Ramalingam and C. M. P. V. S. Sita, "Dimensionality reduced local directional number pattern for face recognition", Journal of Ambient Intelligence and Humanized Computing, Vol.9, No. 1, pp.95-103, 2018.

[16] P. Huang, G. Gao, C. Qian, G. Yang, and Z. Yang, "Fuzzy linear regression discriminant projection for face recognition", IEEE Access, Vol. 5, pp. 4340-4349, 2017.

[17] Z. Jian, Z. Chao, Z. Shunli, L. U. Tingting, S. U. Weiwen, and J. I. A. Jian, "Pre-detection and dual-dictionary sparse representation based face recognition algorithm in non-sufficient training samples", Journal of Systems Engineering and Electronics, Vol.29, No.1, pp.196-202, 2018.

[18] S. Biswas and J. Sil, "An efficient face recognition method using contourlet and curvelet transform", Journal of King Saud University-Computer and Information Sciences, In Press, 2017.

[19] Y. Lu, X. Fang, and B. Xie, "Kernel linear regression for face recognition", Neural Computing and Applications, Vol.24, No.7-8, pp.1843-1849, 2014.

[20] Y. Peng, J. Ke, S. Liu, J. Li, and T. Lei, “An improvement to linear regression classification for face recognition", International Journal of Machine Learning and Cybernetics, pp.1-15, 2018.

[21] S. M. Huang and J. F. Yang, "Linear discriminant regression classification for face recognition", IEEE Signal Processing Letters, Vol.20, No.1, pp.91-94, 2013.

[22] P. Huang, Z. Lai, G. Gao, G. Yang, and Z. Yang, "Adaptive linear discriminant regression classification for face recognition", Digital Signal Processing, Vol.55, pp.78-84. 2016.

[23] K. Reddy and P. Kiran, "Kernel Linear Collaborative Discriminant Regression Classification for Face Recognition Using Local Binary Pattern", Journal of Theoretical \& Applied Information Technology, Vol.95, No.16, 2017.

[24] X. Qu, S. Kim, R. Cui, and H. J. Kim, "Linear collaborative discriminant regression classification for face recognition", Journal of Visual Communication and Image Representation, Vol.31, pp.312-319, 2015. 